\title{
Epidemiología del cáncer de vejiga con énfasis en Colombia - revisión de la literatura
}

\section{Epidemiology of Bladder Cancer with Emphasis in Colombia - Review of the Literature}

\author{
Mauricio Medina-Rico ${ }^{1}$ Manuel Lobo ${ }^{2}$ Jorge Romo ${ }^{2}$ Juan Guillermo Prada ${ }^{2}$ Hugo López-Ramos ${ }^{3}$ \\ ${ }^{1}$ Departamento de Epidemiología Clínica y Bioestadística, Facultad de \\ Medicina, Pontificia Universidad Javeriana, Bogotá, Colombia \\ Address for correspondence Mauricio Medina-Rico, MD, MSc, \\ 2 Estudiante de 5 to año de Medicina, Pontificia Universidad Javeriana, \\ Bogotá, Colombia \\ ${ }^{3}$ Departamento de Urología, Facultad de Medicina, Pontificia

Universidad Javeriana, Bogotá, Colombia

\section{Resumen}

Palabras Clave

- cáncer de vejiga

- epidemiología

- incidencia

- prevalencia

- mortalidad

- sobrevida

\section{Abstract}

Introducción El cáncer de vejiga es el noveno cáncer más frecuente para ambos sexos con una incidencia en ascenso. Habiendo una aparente relación entre el desarrollo de los países y la epidemiología del cáncer; el objetivo de este artículo es observar la epidemiología del cáncer de vejiga en nuestro medio respecto a otros países y describir los posibles factores asociados.

Métodos Se realizó una revisión de la literatura en cuatro bases de datos: Medline, Embase, Cochrane database y Lilacs. Se incluyeron estudios de incidencia, prevalencia, mortalidad, sobrevida y carga de enfermedad, publicados en los últimos 5 años.

Resultados Se encontraron 7806 referencias que fueron discriminadas por título y resumen, incluyendo al final 44 referencias en texto completo. Se observaron tasas de incidencia y mortalidad diferentes, siendo las más altas las encontradas en Europa principalmente en la República Checa seguido de Norte América. En Colombia, la incidencia general es de 6,8/100.000 en hombres y 2,1/100.000 en mujeres entre 2003 y 2007, encontrando cifras bajas en departamentos como la Guajira.

Conclusiones Se ha observado un ascenso en la incidencia y mortalidad del cáncer de vejiga a nivel global. Parece haber una asociación con el tabaquismo y la dieta, así como con el Índice de Desarrollo Humano. Existe una escasa información en Latino América. En Colombia, observando los datos de algunos departamentos y la distribución de especialistas por regiones, se puede suponer un sub-registro haciendo necesario mejorar los sistemas de vigilancia, así como las políticas de salud para el diagnóstico e intervención temprana del cáncer.

Introduction Bladder cancer is the ninth most common cancer for both sexes with an increasing incidence. There is an apparent relationship between the development of countries and the epidemiology of cancer; the objective of this article is to observe the received

September 15, 2017

accepted

March 9, 2018
DOI https://doi.org/ $10.1055 / \mathrm{s}-0038-1645846$. ISSN 0120-789X. eISSN 2027-0119.
Copyright (c) 2019, Sociedad Colombiana License terms de Urología. Publicado por Thieme Revinter Publicações Ltda., Rio de Janeiro, Brazil. Todos los derechos reservados.

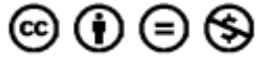



Keywords
- bladder cancer
- epidemiology
- incidence
- prevalence
- mortality
- survival rate

epidemiology of bladder cancer in our environment respect to other countries and to describe the possible associated factors.

Methods A review of the literature was performed in four databases: Medline, Embase, Cochrane database and Lilacs. Studies about Incidence, prevalence, mortality, survival and burden of disease, published in the past 5 years, were included. Results 7806 references were found that were discriminated by title and abstract, 44 references in full text were included at the end. Different incidence and mortality rates were observed, the highest being found in Europe mainly in the Czech Republic followed by North America. In Colombia, the general incidence was 6.8 / 100,000 in men and 2.1 / 100,000 in women between 2003 and 2007, finding low numbers in departments such as Guajira.

Conclusions There has been an increase in the incidence and mortality of bladder cancer worldwide. There seems to be an association with smoking and diet, as well as with the Human Development Index. There is little information in Latin America; in Colombia, observing the data of some departments and the distribution of specialists in the different regions a sub-register can be consider, making necessary to improve surveillance systems, as well as health policies for the diagnosis and early intervention of cancer.

\section{Introducción}

El cáncer de vejiga (CV), es el noveno cáncer más frecuente para ambos sexos con un estimado de 429.000 casos nuevos para el 2012, así como un estimado de 165.000 muertes en ese año según las cifras de GLOBOCAN ${ }^{1}$; haciendo de él la 14va causa más frecuente de muerte por cáncer a nivel global. ${ }^{2}$ Las tasas de incidencia más altas se encuentran en países desarrollados principalmente Europa; por el contrario, la mortalidad siendo ligeramente mayor en países en desarrollo ejemplo en África del norte y medio oriente. ${ }^{3}$ Similar a otros cánceres, la incidencia y mortalidad del CV han tenido un importante ascenso a nivel global. Lo anterior asociado a una mayor exposición de factores de riesgo y principalmente a mejores programas de registro de enfermedad. ${ }^{4}$

Algunos factores de riesgo asociados al CV descrito en la literatura son: predisposición genética, sexo y edad, siendo más prevalente en hombres y adultos mayores de 60 años aumentando más el riesgo después de los 70 años. ${ }^{5}$ Así mismo, se han venido estudiando otros factores asociados al desarrollo de la enfermedad como: consumo de tabaco, polución, dieta, comorbilidades médicas como la diabetes y factores de exposición tales como el trabajo en industrias de derivados del petróleo, fundidoras y productoras de pintura., ${ }^{4,6}$

Para el manejo del CV, en Colombia contamos con una guía de manejo la cual contempla las intervenciones en las diferentes etapas del cáncer.7 Epidemiológicamente, según las estadísticas de GLOBOCAN 2012 estimadas para el periodo comprendido entre 2007 y 2011 por el Instituto Nacional de Cancerología, la tasa de incidencia estandarizada por edad calculada para $\mathrm{CV}$ fue de 4,5/100.000 hombres y 1,3/100.000 en mujeres, con una mortalidad ajustada por edad de 1,4/100.000 en hombres y $0,6 / 100.000$ en mujeres. ${ }^{8}$
La mayoría de los estudios realizados en otros países, se desarrollan a partir de datos epidemiológicos obtenidos de bases poblacionales de cáncer. Colombia, por su parte, posee la base de datos más antigua de América Latina con el registro poblacional de cáncer de Cali (RPCC), que contiene información desde $1962 .{ }^{9}$ Sin embargo, dado las diferencias geográficas y socio-culturales de nuestro país la información del RPCC es insuficiente para calcular la conducta epidemiológica nacional. $^{10}$

Dada la falta de datos epidemiológicos precisos y recientes del CV en Colombia, se hace necesario realizar una búsqueda en la literatura para describir la incidencia, prevalencia y mortalidad de esa enfermedad a lo largo del país, y observar cómo se encuentran esos datos en comparación con otros países, tanto de la región latinoamericana como del resto del mundo y describir cuáles han sido los posibles factores de riesgo asociados a las posibles diferencias.

\section{Métodos}

Se realizó una revisión de la literatura en 4 bases de datos (PubMed, Embase, Cochrane database y Lilacs) y se recogió información de un documento sobre datos publicados en la página del Instituto Nacional de Cancerología con el fin de ampliar la información de los datos nacionales existentes.

Se establecieron los criterios de búsqueda en forma de texto libre y términos indexados. Para caracterizar el cáncer de vejiga $(\mathrm{CV})$ se utilizaron los términos libres: "Bladder cancer," "Bladder neoplasm," "Bladder neoplasia." Para estudios epidemiológicos se incluyeron los términos en texto libre: "incidence," "prevalence," "epidemiology," "mortality," "burden of disease," "cost of illness" y los términos indexados: "Incidence," y "Prevalence." Como 
restricción en la búsqueda se limitó a publicaciones en los últimos 5 años.

Los artículos debían ser estudios originales, que aportaran información epidemiológica de incidencia, prevalencia, mortalidad, sobrevida y carga de la enfermedad para CV. Los estudios destinados a encontrar dicha información en subgrupos específicos (únicamente para un tipo histológico, o un grupo étnico específico) fueron excluidos. Las referencias fueron revisadas por título y resumen por dos revisores de forma independiente. A partir de la primera selección de artículos, se revisaron las referencias en texto completo asegurando que ellos dieran algún dato de interés mencionado previamente del CV para la elaboración del estudio. Se eliminaron los artículos duplicados, y se ignoraron los estudios que estuvieran escritos en un idioma diferente al inglés o al español. Posteriormente se analizó la información obtenida, dando prioridad a los datos correspondientes a Colombia y la región de América Latina.

\section{Resultados}

La búsqueda arrojó un total de 7806 referencias, que se revisaron por título y resumen. En total, se escogieron 346 referencias para revisión en texto completo, de las cuales se excluyeron 303, dado que no expresaban datos epidemiológicos de interés, por reportar informaciones de un grupo etario específico o un único tipo histológico. Las referencias que no tenían disponible texto completo (formato de poster o resumen), fueron excluidas, así como aquellas que estuvieran en un lenguaje diferente al inglés o al español. Se analizaron finalmente 44 artículos en texto completo para la extracción de datos, de los cuales 7 estudios contienen información latinoamericana (-Fig. 1).

Entre los estudios seleccionados, 3 incluían datos globales, 13 evaluaron poblaciones en Europa, 12 en Asia, 4 en África y medio oriente y 12 en América de los cuales 5 corresponden a la literatura de América Latina.

A nivel global se encontraron tres estudios. En el estudio de Mahdavifar N y col. encontraron que la incidencia más alta se encuentra en Bélgica con 17,5/100.000, seguido de Líbano, Malta, Turquía, y Dinamarca, a su vez muestra la mortalidad más alta en Turquía con 6,6/100.000 habitantes, seguido de Egipto, Iraq, Líbano y Mali. ${ }^{3}$ Por otra parte, Ferlay J y col., evidenciaron una incidencia una incidencia global de 9,2/ 100.000 y 3,0/100.000 personas para hombres y mujeres respectivamente para el 2012, con una prevalencia en hombres de 330/100.000 y en mujeres de 99,4/100.000 habitantes y una mortalidad en hombres de 3,2/100.000 y en mujeres de 0,9/100.000 habitantes. ${ }^{1}$ Por último, Crocetti Ey col. mostraron que la incidencia más alta la tiene Italia con 50/ 100.000 habitantes seguida por USA, países Nórdicos, Francia y Australia; Igualmente, comentan las limitaciones que presentan las bases de datos de Italia y los países Nórdicos dada la utilización de diferentes métodos diagnósticos y registro entre las bases de datos de los diferentes países (-Tabla 1). ${ }^{11}$

\section{Europa}

Cinco estudios evaluaron múltiples países. En el estudio realizado por Ferlay y col., encontraron una incidencia general

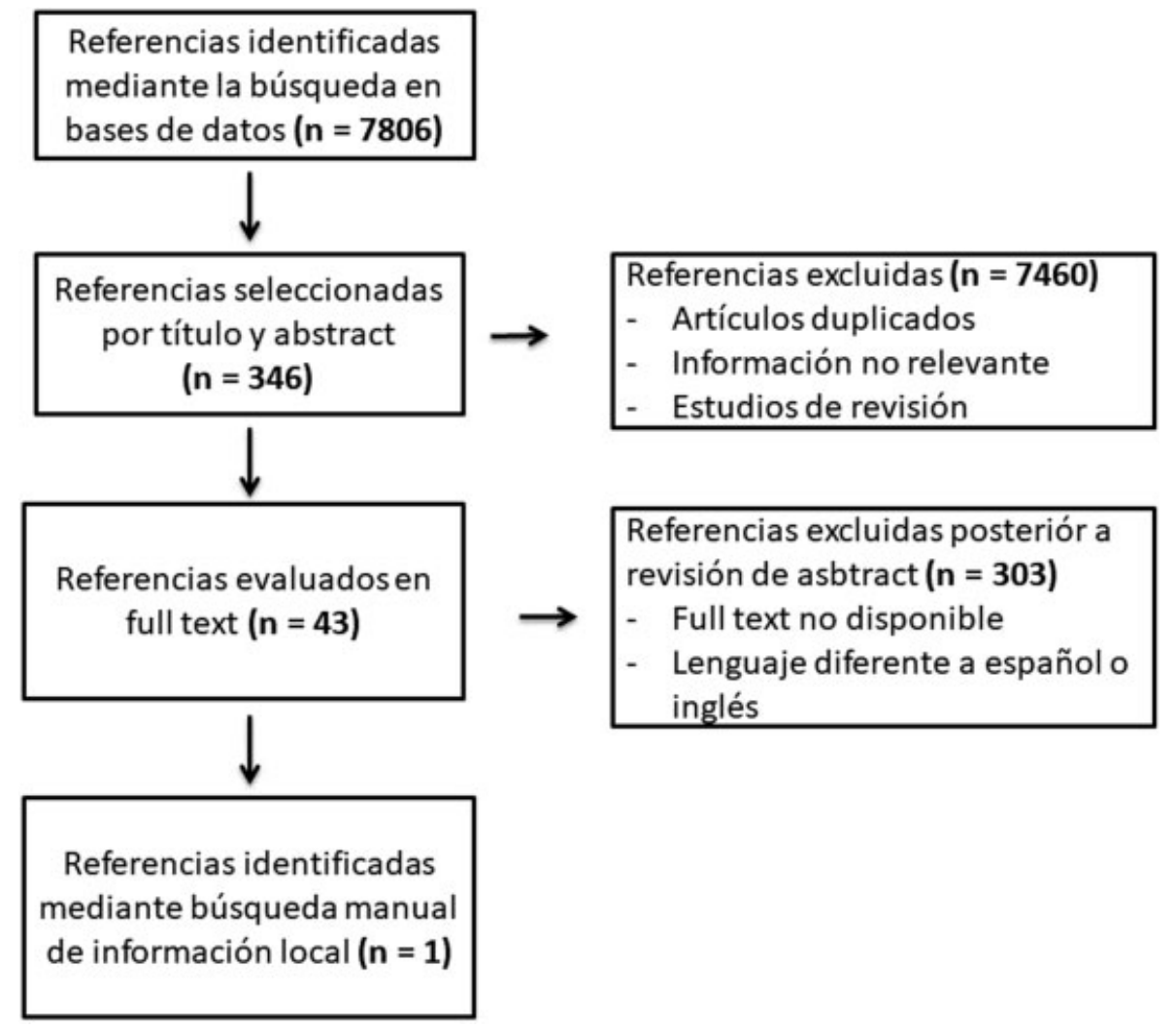

Fig. 1 Flujograma de la búsqueda. 


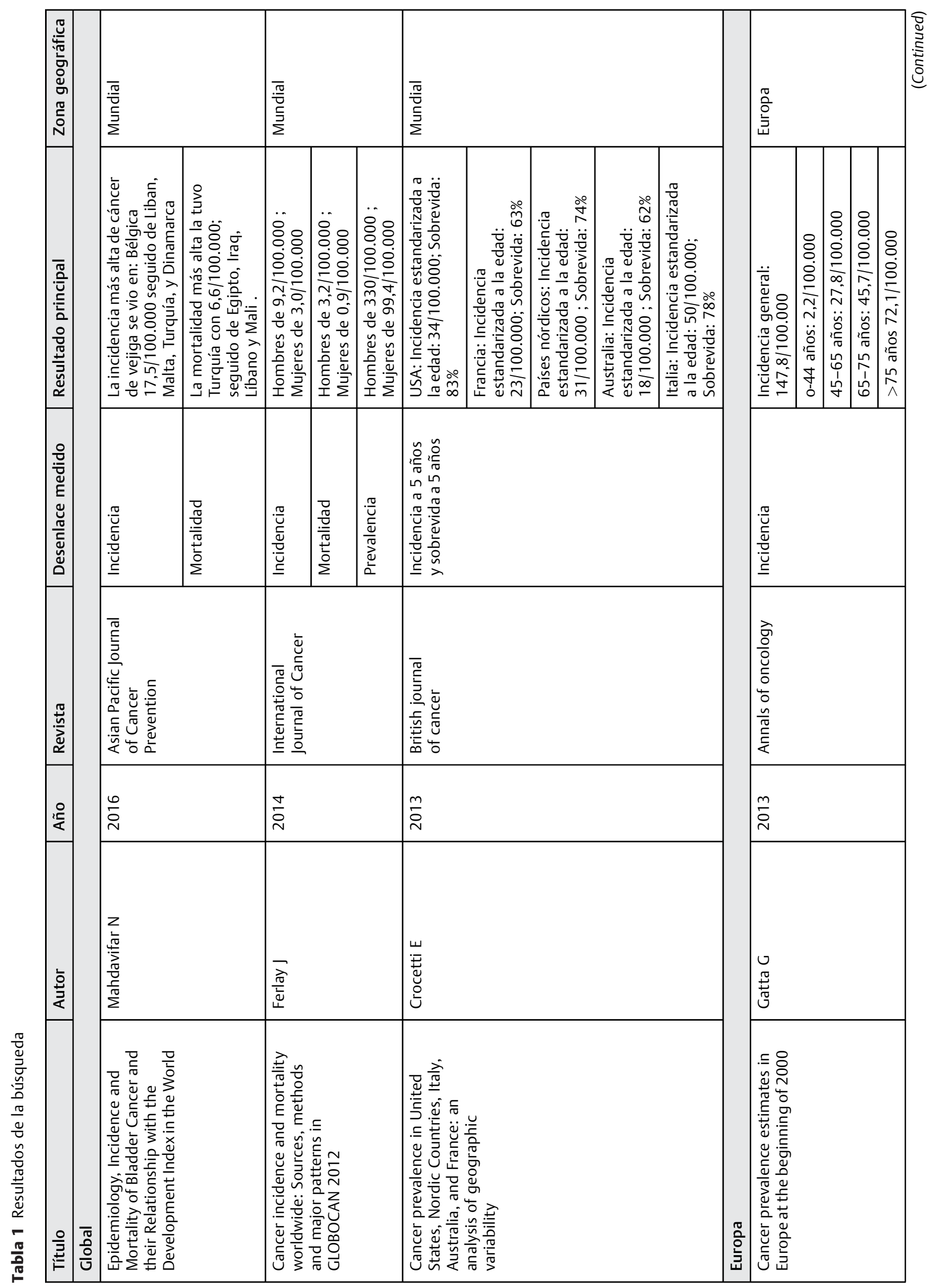




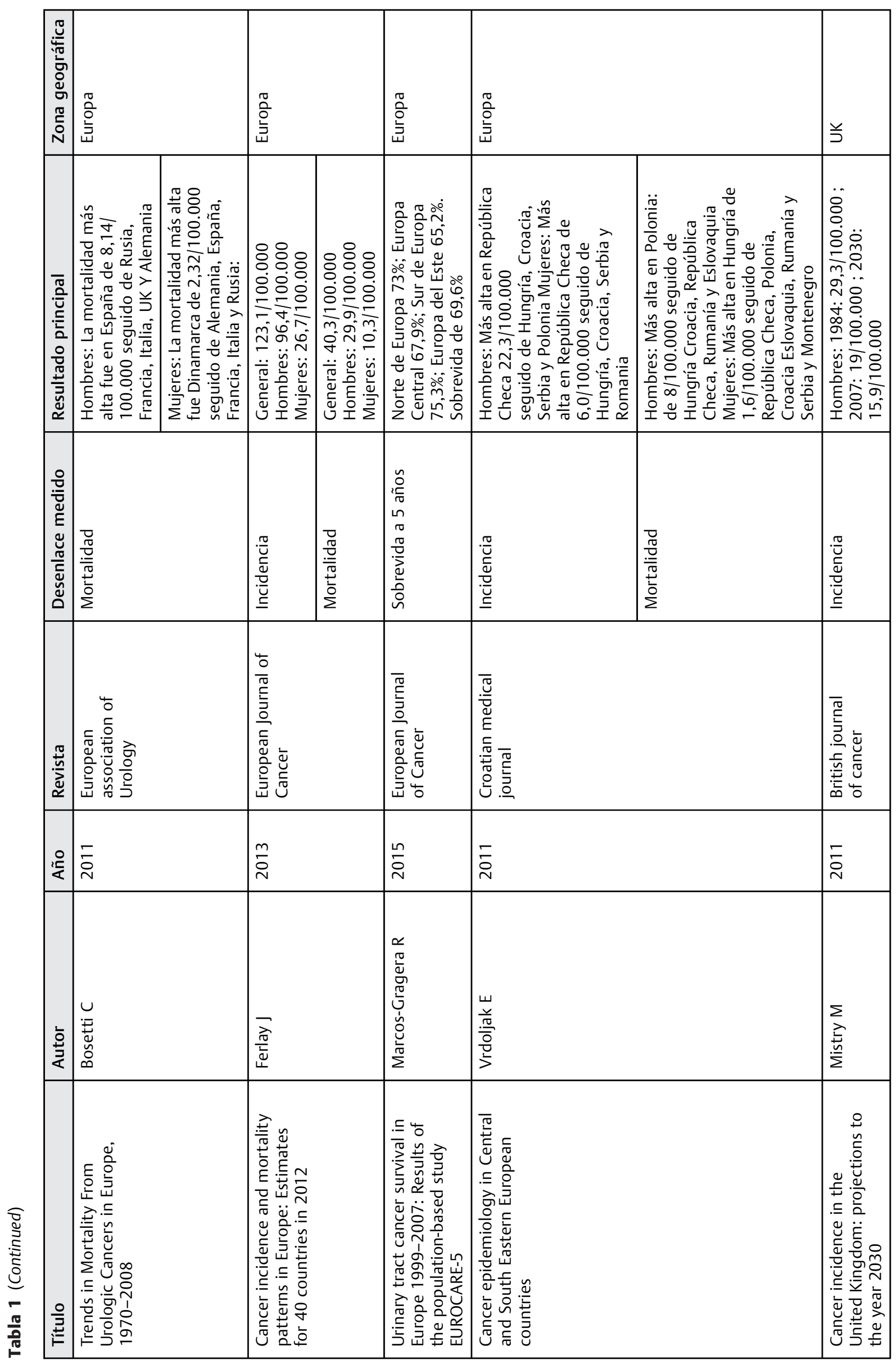




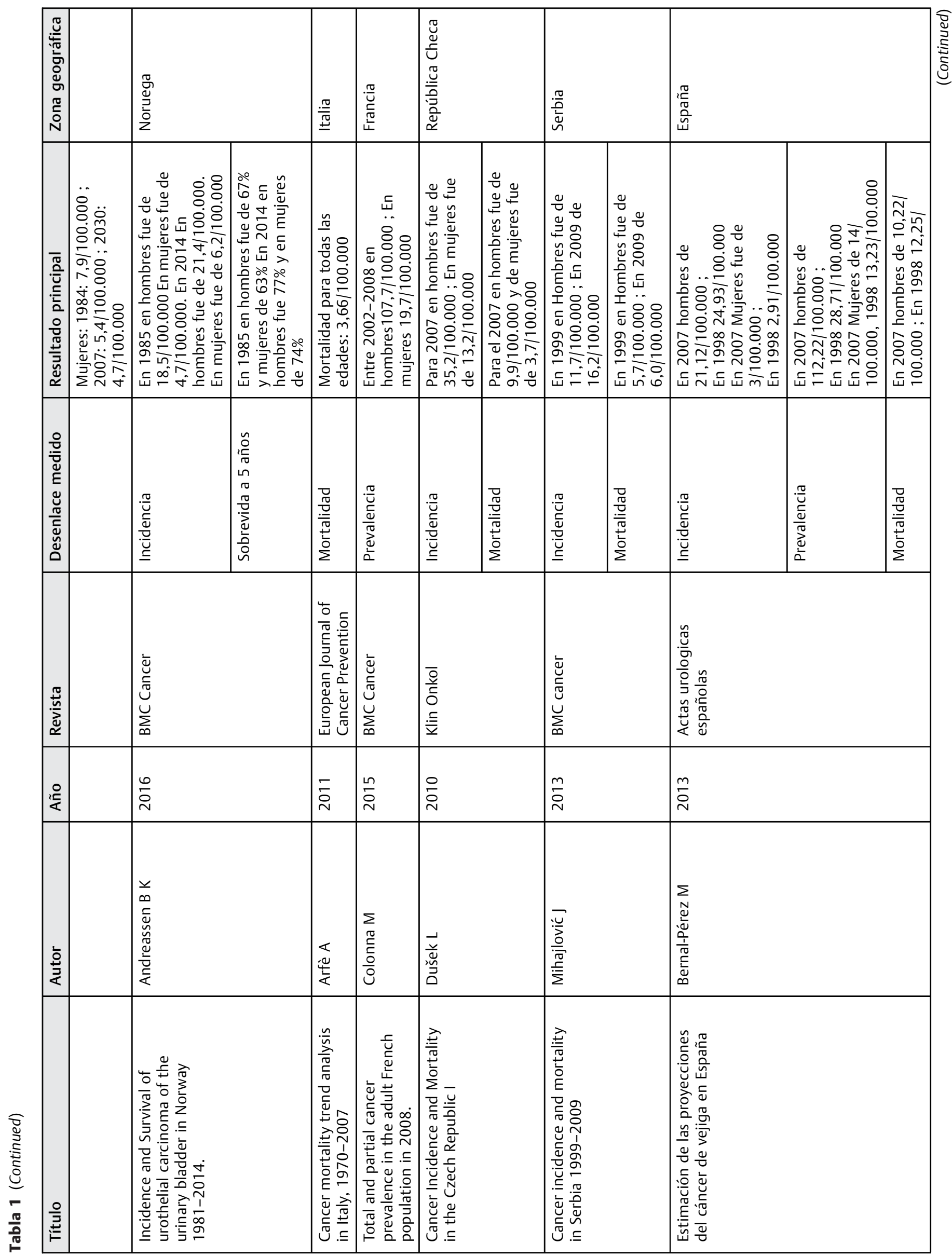




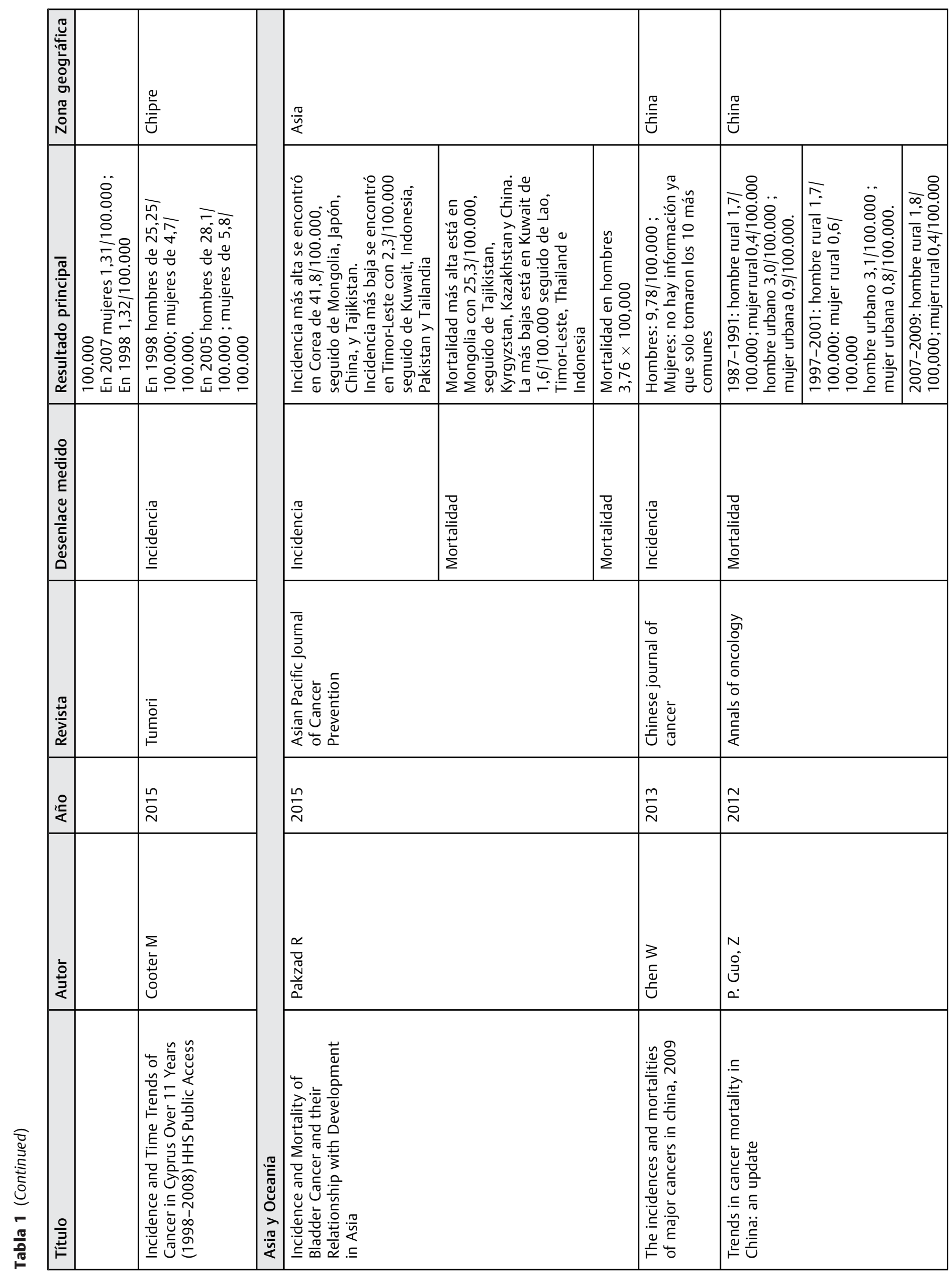




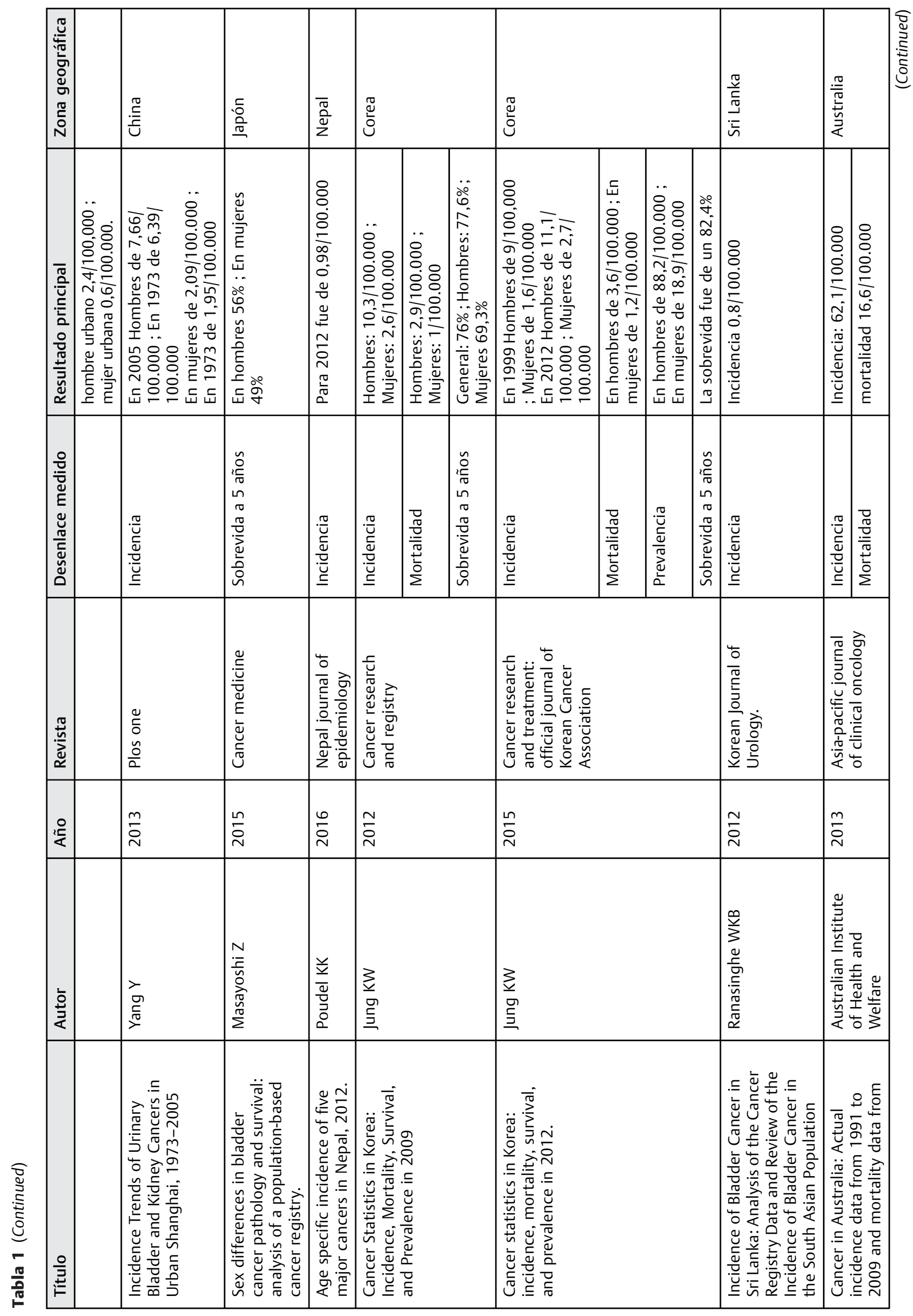




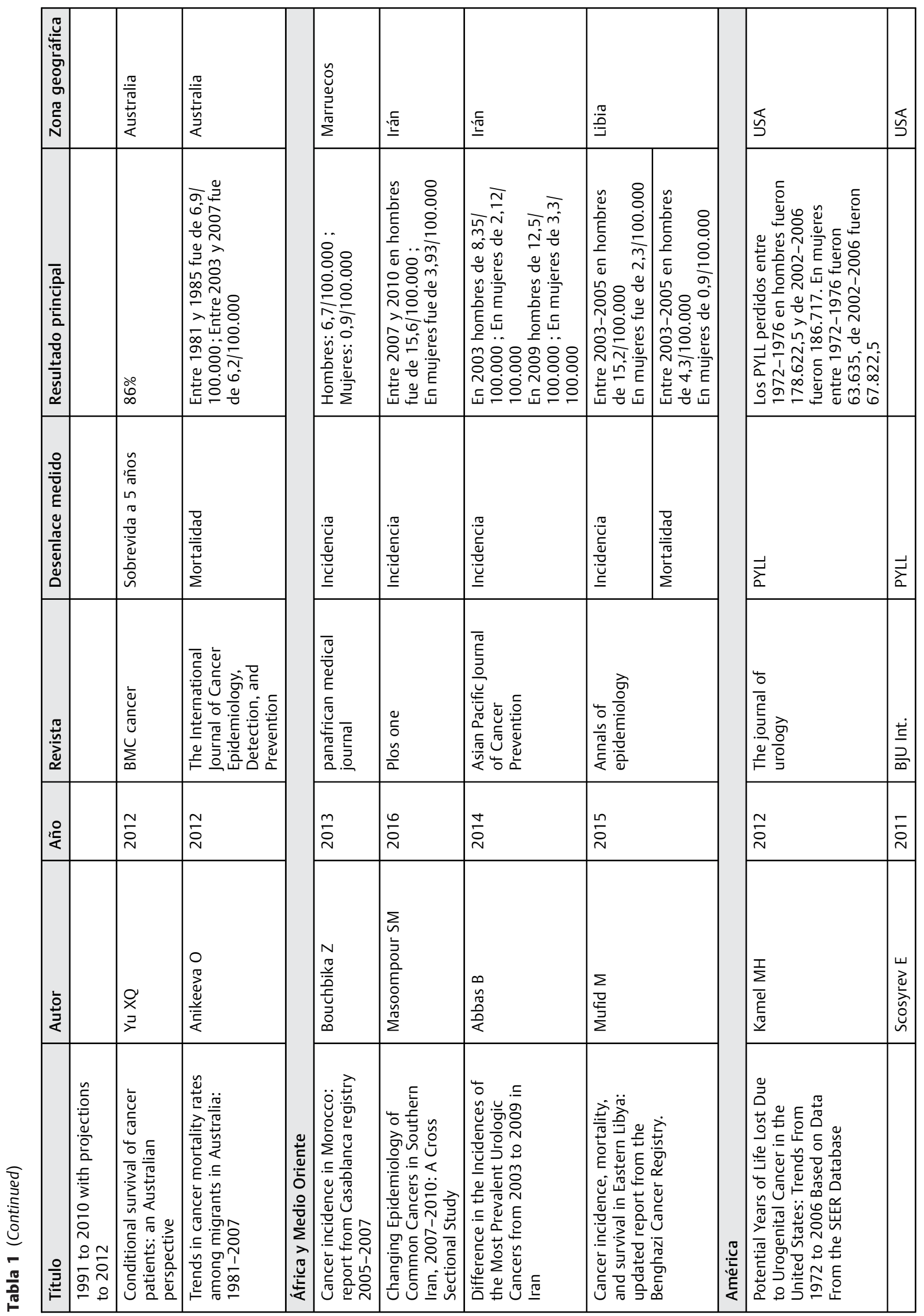




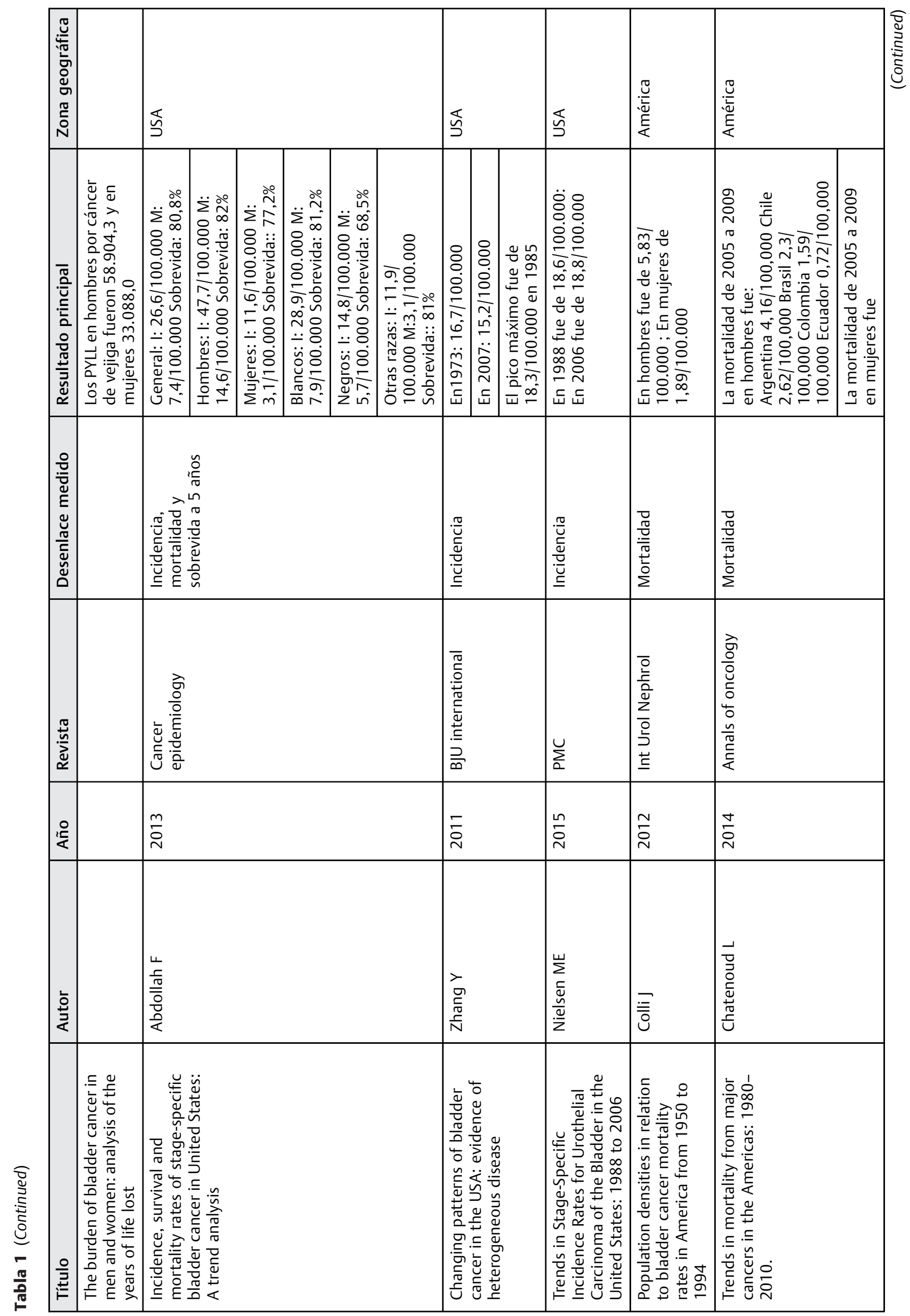




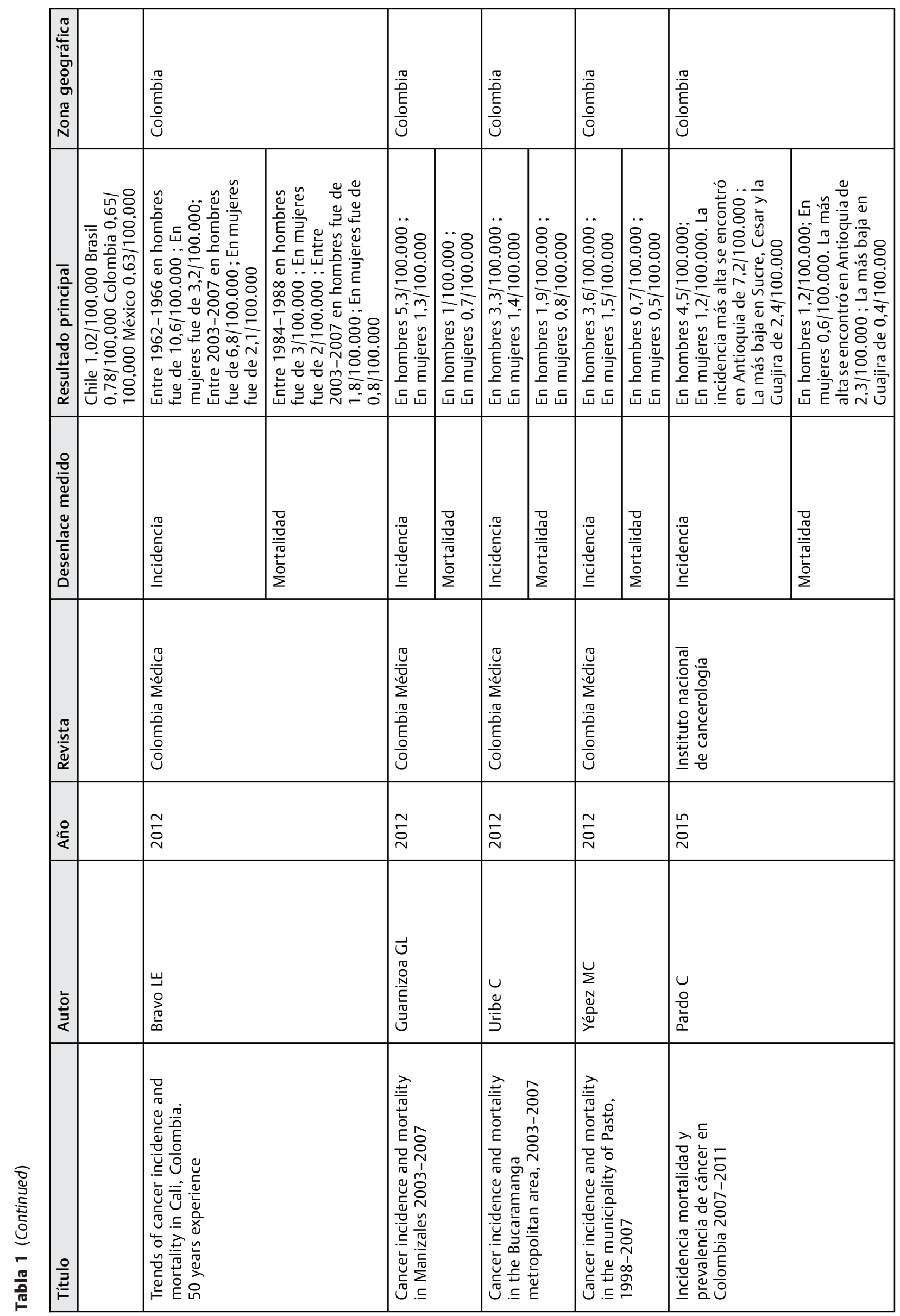


de cáncer de vejiga de 96,4/100.000 en hombres y 26,7/100.000 en mujeres. ${ }^{12}$ De la misma manera, Vrdoljak y col., encontraron la incidencia más alta para el CV en República Checa tanto para hombres como para mujeres ${ }^{13}$; Por su parte, Bosetti y col., evidenciaron la mortalidad más alta en España para hombres y en Dinamarca para mujeres de 8,14/100.000 y 2,32/100.000 respectivamente. $^{14}$ Finalmente, Marcos-Gragera y col., describieron una sobrevida a 5 años para Europa del 73\%, siendo más alta en la región del Sur de Europa en comparación con Europa Central y Europa del Este. ${ }^{15}$ Otros estudios como el de Gatta y col. evidenció una diferencia en la incidencia por grupos de edad, siendo la incidencia más alta en el grupo de mayores de 75 años con una incidencia general de 72,1/100.000; además, concluyen que gracias a la mejoría de métodos diagnósticos, ha ido disminuyendo la mortalidad y así mismo aumentando la prevalencia de la enfermedad. ${ }^{16}$

Los estudios restantes se realizaron en Noruega, Italia, Francia, República Checa, Serbia, Chipre, España y el Reino Unido para un total de 8 estudios. La incidencia más alta se encontró en la República Checa siendo de 35,2/100.000 para hombres y 13,2/100.000 en mujeres para el 2007. ${ }^{17}$ La más baja se encontró en Serbia de 16,2/100.000 en hombres para el 2009. ${ }^{18}$ En cuanto la incidencia 2 estudios los desarrollados por Bernal-Perez $\mathrm{M}$ y col. y Mistry $\mathrm{M}$ y col. encontraron un descenso de la incidencia a través de los años, ese último llegando a hacer un estimado de la incidencia de 29,3/100.000 personas en 1984 a 15,9/100.000 para el 2030.4,19 Por otro lado, tres estudios concuerdan en un aumento de la incidencia a lo largo de los años Cooter y col. observaron un aumento de 25,2/100.000 personas en 1998, a 28,1/100.000 personas para 2005 para hombres ${ }^{20}$; Así mismo, los estudios de Mihajlović y col. y Andreassen y col., llegaron a hallazgos similares los cuales concluyen que el aumento de la incidencia puede deberse a cambios en los factores de riesgo principalmente el consumo de cigarrillo. ${ }^{18,21}$

La mortalidad se evaluó en cuatro estudios. Mihajlović $\mathrm{J}$ y col. encontraron un aumento de 5,7/100.000 en 1999 a 6,0/100.000 para el 2009 lo cual asocia al retraso de un adecuado diagnóstico, así como programas deficientes de tamizaje para esa enfermedad. ${ }^{18}$ No obstante, los estudios de Bernal-Pérez y col. y Arfè y col. evidenciaron una disminución de la mortalidad, ese último asociándolo a una intervención más oportuna de factores de riesgo tales como tabaco, infecciones urinarias y cambios en la dieta. ${ }^{4,22}$

La prevalencia fue evaluada en dos estudios. Colonna y col., encontraron una prevalencia en Francia de 107,7/100.000 para hombres y 19,7/100.000 personas para mujeres entre el 2002 y el $2008 .^{23}$ Finalmente la sobrevida a 5 años, fue evaluada en seis artículos, dos fueron realizados en múltiples países, Marcos-Gragera y col. encontraron diferencias por regiones siendo la mayor en Europa Central de 64,6\% y la más baja en Europa del Norte de 55,8\%, dicho contraste por diferencias en los programas de diagnóstico e intervención de cada país. ${ }^{15}$ Los otros cuatro estudios fueron realizados en Irlanda, Dinamarca y Escocia, siendo ese último el país con sobrevida más baja 39 y $42 \% .{ }^{24}$ Dentro de los factores asociados, se encontró que un diagnóstico temprano e intervención oportuna, así como el nivel socioeconómico y educativo pueden estar relacionados con las diferencias epidemiológicas del cáncer. ${ }^{25}$ Por su parte, Engholm G y col. describieron una mayor sobrevida en hombres que en mujeres. ${ }^{26}$

\section{África y Oriente Medio}

Se encontraron 4 estudios pertenecientes a Marruecos, Irán, y Libia. El estudio de Bouchbika $Z$ y col. realizado en Marruecos, encontró una incidencia mayor en hombres que mujeres, siendo 6,7/100.000 en hombres y 0,9/100.000 en mujeres. ${ }^{27,28}$ Los estudios de Abbas B y col. y Masoompour y col. realizados en Irán encontraron hallazgos similares en la distribución por sexos; sin embargo, Abbas B y col. encontraron además un aumento de la incidencia en el tiempo de 8,3/100.000 en hombres y 2,1/100.000 en mujeres para 2003, y 12,5/100.000 en hombres y 3,3/100.000 en mujeres para 2009. ${ }^{29,30}$ Finalmente, Mufid D y col. en Libia, fuera de evaluar incidencia, observaron mortalidad encontrando una tasa de 4,3/100.000 en hombres y 0,9/100.000 en mujeres. ${ }^{31}$

\section{Asia y Oceanía}

Un total de 12 estudios se obtuvieron. Un estudio hecho por Pakzad $\mathrm{R}$ y col. involucró todo el continente asiático, encontrando la incidencia más alta en Corea con 41,8/ 100.000 habitantes, seguido de Mongolia, Japón, China, y Tayikistán. Mientras que la incidencia más baja se encontró en Timor-Leste de 2,3/100.000, seguido de Kuwait, Indonesia, Pakistán y Tailandia. Dichas diferencias en la incidencia posiblemente asociadas al tabaquismo y a la contaminación industrial de cada país. Por su parte, la mortalidad más alta la encontró en Mongolia de 25,3/100.000 habitantes, seguido de Tayikistán, Kirguistán, Kazajistán y China; La mortalidad más baja se dio en Kuwait de 1,6/100.000 habitantes, seguido por Lao, Timor-Leste, Tailandia e Indonesia. ${ }^{32}$

En China se realizaron tres estudios. Chen $\mathrm{W}$ y col., mostraron una incidencia de 6,98/100.000 habitantes. $^{33}$ Dos estudios concluyeron un aumento de la incidencia del cáncer de vejiga con los años pasando de 6,39/100.000 en 1973 a 7,66/100.000 en 2005; adicionalmente se encontró que desde 1973 hasta 2009 hubo un aumento de la mortalidad en áreas rurales y una disminución en áreas urbanas, posiblemente por la exposición ocupacional que puede presentar la población rural. ${ }^{34,35}$ En Japón, Masayoshi $\mathrm{Z}$ y col. encontraron una sobrevida a 5 años del $56 \%$ para hombres y $49 \%$ para mujeres. ${ }^{36}$ En Nepal, Poudel $\mathrm{K}$ y col. describieron una incidencia de 0,98/100.000, siendo el CV el quinto cáncer más común en el país. ${ }^{37}$

En Corea se realizaron dos estudios, los cuales comparan incidencia, mortalidad y sobrevida a 5 años entre los géneros de la población, ambos realizados por Jung Ky col., los cuales mostraron un aumento de la incidencia de 9/100.000 en hombres y 1,6/100.000 en mujeres para 2009 a 11,1/100.000 en hombres y 2,7/100.000 en mujeres para 2012, lo cual asociaron a un aumento del consumo de cigarrillo en los últimos años; una mortalidad de 3,6 /100.000 en hombres y $1,2 / 100.000$ en mujeres con datos previos que muestran una mortalidad de 2,9/100.000 en hombres y 1,0/100.000 en mujeres y finalmente una sobrevida general a 5 años que 
aumentó de un $76 \%$ a un $82,4 \% .{ }^{38,39}$ En Sri Lanka se realizó un estudio que demostró una incidencia total de 0,8/100.000 habitantes del 2001 al 2005, siendo ese el 15vo cáncer más común en la población; adicionalmente en él se comenta cómo los datos se pueden ver comprometidos ya que hay muchas personas a las que no se les hace diagnóstico. ${ }^{40}$

En Australia se encontraron tres artículos. El estudio realizado por el Australian Institute of Health and Welfare mostró una Incidencia de 62,1/100.000 y una mortalidad de $16,6 / 100.000 .{ }^{41}$ Por otro lado, Xue Qin Yu y col. mostraron una sobrevida a 5 años del $86 \%{ }^{42}$ Finalmente, Anikeeva $O$ y col. evidenciaron una disminución en la mortalidad la cual es más significativa en países con mejores ingresos como el Reino Unido y países del sur y el oeste de Europa. ${ }^{43}$

\section{América}

Se encontraron dos estudios que contenían toda la población de continente, cinco estudios realizados en EE.UU, y cinco estudios Colombianos. El estudio realizado por Abdollah $\mathrm{F}$ y col., encontró que la incidencia en Estados Unidos en hombres es de 47,7/100.000 habitantes y en mujeres es de 14,6/100.000 habitantes. Adicionalmente, ese estudio encontró una mayor incidencia en raza blanca respecto a negros y otras razas. ${ }^{44}$ Dos estudios realizados por Zhang Y y col. y Nielsen ME y col., no encontraron cambios de la incidencia a través del tiempo, el último por ejemplo, evidenciando una incidencia de 18,6/ 100.000 en 1988 y de $18,8 / 100.000$ para $2006 .{ }^{45,46}$ Acerca de los años de vida potencialmente perdidos (PYLL) se encontraron dos estudios de Scosyrev E y col. y Kamel MH y col. ese último encontrando un aumento de los PYLL a través del tiempo de $178.622,5$ en hombres y 63.635 en mujeres entre 1972 y 1976, a 186.717 en hombres y 67.822,5 en mujeres entre 2002 y 2006. ${ }^{47,48}$ Los estudios de Colli J y col. y Chatenoud L y col., encontraron para Latino América la mayor mortalidad en Argentina para hombres de 4,16/100.000 y Chile para mujeres de 1,02/100.000 habitantes, y la menor mortalidad en Ecuador para hombres de 0,72/100.000 y México de 0,63 para mujeres. ${ }^{49,50}$

En Colombia el estudio realizado por Bravo LE y col. reveló un descenso de la incidencia de 10,6/100.000 en hombres y 3,2/100.000 en mujeres entre 1962 y 1966 a 6,8/100.000 hombres y 2,1/100.000 mujeres entre 2003 y $2007 .{ }^{9}$ El resto de estudios que evaluaron mortalidad describen una aparente diferencia entre los datos de los departamentos colombianos, siendo la incidencia más alta en Antioquia de $7,2 / 100.000$ y la más baja en Guajira de 2,4/100.000..$^{8,51-53}$ De manera similar, la mortalidad también descendió con los años de 3/100.000 en hombres y 2/100.000 en mujeres entre 1984 y 1988 a 1,8/100.000 en hombres y 0,8/100.000 en mujeres entre 2003 y $2007^{9}$; igualmente, la mortalidad no varió de manera importante en las regiones siendo más alta en Antioquia de 2,3/100.000 habitantes y la más baja en la Guajira de $0,4 / 100.000$ habitantes. ${ }^{8,51-53}$

\section{Discusión}

Con esta revisión se pudo observar la diferencia global de las tasas de incidencia, mortalidad y sobrevida del CV. La mayor incidencia evidenciándose en Europa, principalmente República Checa; seguido de América del Norte, Asía, Australia, Medio Oriente y Latino América. Igualmente, la tasa de mortalidad se encontró más alta en Europa, y más baja en Latino América. ${ }^{3,49}$ Igualmente, se observó que las tasas epidemiológicas han aumentado, algunos con diferencias aparentemente leves a lo largo de los años, dichos cambios se han dado debido entre otras cosas a fallas en el diagnóstico temprano y tratamiento oportuno de la enfermedad. ${ }^{18,29}$ Así mismo, las diferencias epidemiológicas han podido asociarse a un aumento en la expectativa de vida así como a una mayor exposición a factores de riesgo como tabaco, dieta, entre otros $^{16,30}$; Lo cual requiere ser profundizado en futuros estudios acerca de su papel en el desarrollo del CV.

Se identificó una diferencia por sexo y raza, teniéndose en todos los estudios que compararon dichas poblaciones una tasa de incidencia y mortalidad mayor en hombres respecto mujeres y en las poblaciones blancas respecto negros y otras poblaciones. ${ }^{44}$ Así mismo, estudios como el de Pakzad R y col. o el de Mahdavifar N y col., encontraron una relación de la incidencia con el Índice de Desarrollo Humano (HDI), viéndose una menor incidencia en países con menor desarrollo ${ }^{3,32}$; lo cual puede dar a pensar que la baja incidencia de esos países puede deberse a un menor diagnóstico de la enfermedad. Hallazgos similares pueden verse con la mortalidad en el estudio de Guo PZ y col., donde hay una mayor mortalidad en la región urbana respecto la rural. ${ }^{35}$ Eso es de interés para Colombia y el resto de Latino América, siendo que los países de esta región, tienen una población rural importante, e igualmente son países en su mayoría de ingresos medios. ${ }^{54}$

De los datos encontrados en Colombia y Latino América, se encontró como la incidencia y mortalidad del CV parece ser menor en comparación a Europa y EE.UU. No obstante, el estudio de Uribe $\mathrm{C}$ y col., mencionando las dificultades de detección y registro de enfermedad, ${ }^{52}$ haciendo de esa comparación algo complejo.

El problema de comparación se puede observar en los datos de la IC5 (incidencia de cáncer en 5 continentes), en la que se puede ver el número de casos en cada país, pero más importante se puede evaluar la calidad de los registros por valores como: el de DCO\% que se obtienen de los certificados de defunción, el de MV\% correspondiente a los cánceres registrados con verificación microscópica, y el MI\% que es la relación entre la mortalidad y la incidencia de cáncer en un período dado. Teniendo un estimado para cada valor en cáncer de vejiga, puede ser percibido como que hay un mayor número de casos reportados por certificados de defunción en América Latina respecto a América del norte o Europa, así como un menor número de casos confirmados por microscopio, con lo que se podría pensar en una menor calidad en los registros en los países de Latino América.

Uno de los hallazgos más importantes se observa es la aparente diferencia en las tasas epidemiológicas de las diferentes regiones del país, siendo bajas en departamentos que se caracterizan por tener menores ingresos como la Guajira. Eso podría asociarse a mayores dificultades en cuanto al acceso a los servicios de salud; además de acceso a 
médicos especialistas que observando la distribución de esos en el país, hacen de la Urología una especialidad prácticamente inexistente en dichos lugares, ${ }^{55}$ lo que se puede asociar en un menor diagnóstico y registro de la enfermedad.

Como fortaleza, esta revisión cuenta con una búsqueda amplia de la literatura en diferentes bases de datos, y una revisión pareada para la extracción de información y una búsqueda reproducible que permite al lector profundizar en el tema si así lo quiere. Dentro de las limitaciones del estudio, tenemos que no es una revisión sistemática y por eso no contamos con toda la evidencia existente, así mismo no pretendemos evaluar la homogeneidad de los artículos, ${ }^{56}$ por lo cual los resultados obtenidos corresponden a una descripción de los hallazgos emanados de los diferentes estudios con una comparación subjetiva de los resultados.

Pese a los hallazgos, debido a la metodología de los estudios revisados los cuales extrajeron su información de bases de datos de cada país, y dado que los programas de registro de enfermedad tienen cobertura y calidad diferente en cada país, no se puede hacer una adecuada comparación de esos resultados ${ }^{11}$; Mihajlović y col. por ejemplo, hacen referencia a una ausencia de información de prevalencia y sobrevida en Serbia. ${ }^{18}$ Así mismo, para la mortalidad en los estudios de Ferlay y col. o Chen $\mathrm{W}$ y col. se comentó la dificultad de certificar las verdaderas causas de muerte de las personas y por ende creando un subestimado de datos. ${ }^{12,33}$ Sin embargo, en términos generales dado el aparente aumento de la incidencia del CV surge la necesidad estudiar la posible implementación de programas de prevención y control de factores de riesgo; así como de tamización y atención temprana, que permita la intervención oportuna de los pacientes con CV.

\section{Conclusión}

Nuestro estudio identifica una aparente tendencia ascendente de la incidencia del CV en la mayoría de países asociado posiblemente a una mayor expectativa de vida siendo este un cáncer principalmente del adulto mayor, así como una mayor presencia de factores de riesgo que merecen atención para futuras investigaciones como: tabaco y dieta. Esta revisión permitió una aproximación a la epidemiología del CV en nuestro medio y su comparación con países de otros continentes. Como hallazgo de interés el nivel socioeconómico y el índice de desarrollo humano regional se identificó como posible factor asociado a las diferencias epidemiológicas en los distintos países, principalmente por la alteración que produce en los programas de detección y registro de la enfermedad. Ante el aumento de la incidencia del CV a nivel global y un descenso aparente en Colombia, tenemos que preguntarnos cómo estamos realizando la detección y el registro del cáncer y de ser el caso, proponer soluciones para mejorar las políticas de salud pública dirigidas a realizar un diagnóstico, registro y tratamiento oportuno a los pacientes con CV en nuestra región.

\section{Consideraciones Éticas}

Protección de sujetos humanos y animales. Los autores declaran que no se realizaron experimentos en seres humanos o animales para este estudio.
Confidencialidad de los datos. No se aplica

Financiación

Ninguna.

Conflicto de Intereses

Ninguno.

\section{Bibliografía}

1 Ferlay J, Soerjomataram I, Dikshit R, et al. Cancer incidence and mortality worldwide: sources, methods and major patterns in GLOBOCAN 2012. Int J Cancer 2015;136(05):E359-E386

2 Ferlay J, Randi G, Bosetti C, et al. Declining mortality from bladder cancer in Europe. BJU Int 2008;101(01):11-19

3 Mahdavifar N, Ghoncheh M, Pakzad R, Momenimovahed Z, Salehiniya H. Epidemiology, Incidence and Mortality of Bladder Cancer and their Relationship with the Development Index in the World. Asian Pac J Cancer Prev 2016;17(01):381-386

4 Bernal-Pérez M, Souza DL, Romero-Fernández FJ, Gómez-Bernal G, Gómez-Bernal FJ. Estimation of bladder cancer projections in Spain. Actas Urol Esp 2013;37(05):286-291

5 Ljungberg B, Campbell SC, Choi HY, et al. The epidemiology of renal cell carcinoma. Eur Urol 2011;60(04):615-621

6 Burger M, Catto JWF, Dalbagni G, et al. Epidemiology and risk factors of urothelial bladder cancer. Eur Urol 2013;63(02):234-241

7 Citarella D, Quiroga Matamoros W, Fernandez F, Patiño I, Estrada A. Guía de manejo en cáncer vesical.Urol Colomb 2016;25(02):154-168

8 Pardo C, Cendales R. Incidencia, mortalidad y prevalencia de Cáncer en Colombia 2007-2011. Inst Nac Cancerol 2015;1:36-37

9 Bravo LE, Collazos T, Collazos P, García LS, Correa P. Trends of cancer incidence and mortality in Cali, Colombia. 50 years experience. Colomb Med (Cali) 2012;43(04):246-255

10 Piñeros M, Ferlay J, Murillo R. Cancer incidence estimates at the national and district levels in Colombia. Salud Publica Mex 2006; 48(06):455-465

11 Crocetti E, De Angelis R, Buzzoni C, et al; AIRTUM Working group. Cancer prevalence in United States, Nordic Countries, Italy, Australia, and France: an analysis of geographic variability. Br J Cancer 2013;109(01):219-228

12 Ferlay J, Steliarova-Foucher E, Lortet-Tieulent J, et al. Cancer incidence and mortality patterns in Europe: estimates for 40 countries in 2012. Eur J Cancer 2013;49(06):1374-1403

13 Vrdoljak E, Wojtukiewicz MZ, Pienkowski T, et al; South Eastern European Research Oncology Group. Cancer epidemiology in Central, South and Eastern European countries. Croat Med J 2011;52(04):478-487

14 Bosetti C, Bertuccio P, Chatenoud L, Negri E, La Vecchia C, Levi F. Trends in mortality from urologic cancers in Europe, 1970-2008. Eur Urol 2011;60(01):1-15

15 Marcos-Gragera R, Mallone S, Kiemeney LA, et al; EUROCARE-5 Working Group. Urinary tract cancer survival in Europe 19992007: Results of the population-based study EUROCARE-5. Eur J Cancer 2015;51(15):2217-2230

16 Gatta G, Mallone S, van der Zwan JM, Trama A, Siesling S, Capocaccia R; EUROCARE Working Group. Cancer prevalence estimates in Europe at the beginning of 2000. Ann Oncol 2013; 24(06):1660-1666

17 Dusek L, Muzik J, Maluskova D, et al. Cancer incidence and mortality in the Czech Republic. Klin Onkol 2014;27(06): 406-423

18 Mihajlović J, Pechlivanoglou P, Miladinov-Mikov M, Zivković S, Postma MJ. Cancer incidence and mortality in Serbia 1999-2009. BMC Cancer 2013;13(01):18

19 Mistry M, Parkin DM, Ahmad AS, Sasieni P. Cancer incidence in the United Kingdom: projections to the year 2030. Br J Cancer 2011; 105(11):1795-1803 
20 Cooter M, Soliman AS, Pavlou P, et al. Incidence and time trends of cancer in Cyprus over 11 years (1998-2008). Tumori 2015;101 (01):8-15

21 Andreassen BK, Aagnes B, Gislefoss R, Andreassen M, Wahlqvist R. Incidence and Survival of urothelial carcinoma of the urinary bladder in Norway 1981-2014. BMC Cancer 2016;16(01): 799

22 Arfè A, Malvezzi M, Bertuccio P, Decarli A, La Vecchia C, Negri E. Cancer mortality trend analysis in Italy, 1970-2007. Eur J Cancer Prev 2011;20(05):364-374

23 Colonna M, Mitton N, Bossard N, Belot A, Grosclaude P; French Network of Cancer Registries (FRANCIM). Total and partial cancer prevalence in the adult French population in 2008. BMC Cancer 2015;15(01):153

24 Westlake S, Cooper N, Rachet B, Coleman MP. Survival from cancers of the kidney and ureter in England and Wales up to 2001. Br J Cancer 2008;99(Suppl 1):S93-S95

25 Pelant T, Larsen EH, Lund L, et al. Survival of patients with kidney cancer in central and northern Denmark, 1998-2009. Clin Epidemiol 2011;3(Suppl 1):53-58

26 Engholm G, Hakulinen T, Gislum M, et al. Trends in the survival of patients diagnosed with kidney or urinary bladder cancer in the Nordic countries 1964-2003 followed up to the end of 2006. Acta Oncol 2010;49(05):655-664

27 Bouchbika Z, Haddad H, Benchakroun N, et al. Cancer incidence in Morocco: report from Casablanca registry 2005-2007. Pan Afr Med J 2013;16:31

28 Tazi MA, Er-Raki A, Benjaafar N. Cancer incidence in Rabat, Morocco: 2006-2008. Ecancermedicalscience 2013;7:338

29 Masoompour SM, Lankarani KB, Honarvar B, Tabatabaee SH, Moghadami M, Khosravizadegan Z. Changing Epidemiology of Common Cancers in Southern Iran, 2007-2010: A Cross Sectional Study. PLoS One 2016;11(05):e0155669

30 Basiri A, Shakhssalim N, Jalaly NY, Miri HH, Partovipour E, Panahi $\mathrm{MH}$. Difference in the incidences of the most prevalent urologic cancers from 2003 to 2009 in Iran. Asian Pac J Cancer Prev 2014;15 (03):1459-1463

31 El Mistiri M, Salati M, Marcheselli L, et al. Cancer incidence, mortality, and survival in Eastern Libya: updated report from the Benghazi Cancer Registry. Ann Epidemiol 2015;25(08): 564-568

32 Pakzad R, Mohammadian-Hafshejani A, Mohammadian M, et al. Incidence and Mortality of Bladder Cancer and their Relationship with Development in Asia. Asian Pac J Cancer Prev 2015;16(16): 7365-7374

33 Chen $\mathrm{W}$, Zheng R, Zhang S, et al. The incidences and mortalities of major cancers in China, 2009. Chin J Cancer 2013;32(03):106-112

34 Yang Y, Xie L, Zheng J-L, Tan Y-T, Zhang W, Xiang Y-B. Incidence trends of urinary bladder and kidney cancers in urban Shanghai, 1973-2005. PLoS One 2013;8(12):e82430

35 Guo P, Huang ZL, Yu P, Li K. Trends in cancer mortality in China: an update. Ann Oncol 2012;23(10):2755-2762

36 Zaitsu M, Toyokawa S, Tonooka A, et al. Sex differences in bladder cancer pathology and survival: analysis of a population-based cancer registry. Cancer Med 2015;4(03):363-370

37 Poudel KK, Huang Z, Neupane PR. Age specific incidence of five major cancers in Nepal, 2012. Nepal J Epidemiol 2016;6(02): 565-573
38 Jung K-W, Park S, Kong H-J, et al. Cancer statistics in Korea: incidence, mortality, survival, and prevalence in 2009. Cancer Res Treat 2012;44(01):11-24

39 Jung K-W, Won Y-J, Kong H-J, et al. Cancer statistics in Korea: incidence, mortality, survival, and prevalence in 2012. Cancer Res Treat 2015;47(02):127-141

40 Ranasinghe WKB, De Silva D, De Silva MVC, et al. Incidence of bladder cancer in sri lanka: analysis of the cancer registry data and review of the incidence of bladder cancer in the South asian population. Korean J Urol 2012;53(05):304-309

41 Australian Institute of Health and Welfare. Cancer in Australia: Actual incidence data from 1991 to 2009 and mortality data from 1991 to 2010 with projections to 2012. Asia Pac J Clin Oncol 2013; 9(03):199-213

42 Yu XQ, Baade PD, O'Connell DL. Conditional survival of cancer patients: an Australian perspective. BMC Cancer 2012;12(01):460

43 Anikeeva O, Bi P, Hiller JE, Ryan P, Roder D, Han G-S. Trends in cancer mortality rates among migrants in Australia: 1981-2007. Cancer Epidemiol 2012;36(02):e74-e82

44 Abdollah F, Gandaglia G, Thuret R, et al. Incidence, survival and mortality rates of stage-specific bladder cancer in United States: a trend analysis. Cancer Epidemiol 2013;37(03):219-225

45 Zhang Y, Zhu C, Curado MP, Zheng T, Boyle P. Changing patterns of bladder cancer in the USA: evidence of heterogeneous disease. BJU Int 2012;109(01):52-56

46 Nielsen ME, Smith AB, Meyer A-M, et al. Trends in stage-specific incidence rates for urothelial carcinoma of the bladder in the United States: 1988 to 2006. Cancer 2014;120(01):86-95

47 Kamel MH, Moore PC, Bissada NK, Heshmat SM. Potential years of life lost due to urogenital cancer in the United States: trends from 1972 to 2006 based on data from the SEER database. J Urol 2012; 187(03):868-871

48 Scosyrev E, Golijanin D, Wu G, Messing E. The burden of bladder cancer in men and women: analysis of the years of life lost. BJU Int 2012;109(01):57-62

49 Chatenoud L, Bertuccio P, Bosetti C, et al. Trends in mortality from major cancers in the Americas: 1980-2010. Ann Oncol 2014;25 (09):1843-1853

50 Colli J, Lee BR, Thomas R. Population densities in relation to bladder cancer mortality rates in America from 1950 to 1994. Int Urol Nephrol 2012;44(02):443-449

51 López Guarnizo G, Arias Ortiz N, Arboleda Ruiz W. Cancer incidence and mortality in Manizales 2003-2007. Colomb Med (Cali) 2012;43(04):281-289

52 Uribe C, Osma S, Herrera V. Cancer incidence and mortality in the Bucaramanga metropolitan area, 2003-2007. Colomb Med (Cali) 2012;43(04):290-297

53 Yépez MC, Bravo LE, HidalgoTroya A, Jurado DM, Bravo LM. Cancer incidence and mortality in the municipality of Pasto, 1998 - 2007. Colomb Med (Cali) 2012;43(04):256-266

54 World Bank Country and Lending Groups - World Bank Data Help Desk. The world bank. Cited on May 15, 2017. Avaliable at: https:// datahelpdesk.worldbank.org/knowledgebase/articles/906519world-bank-country-and-lending-groups

55 Juliao Senior A. Urología Colombiana, Ejercicio Profesional en Ciudades Intermedias. Urol Colomb. 2002;11(03):212-220

56 García-Perdomo HA. Conceptos fundamentales de las revisiones sistemáticas/metaanálisis. Urol Colomb. 2015;24(01):28-34 